\title{
On the Existence of Blow-up Phenomenon of Generalized (2+1) Dimensional Kaup-Kupershmidt Equation
}

\author{
Yeung Ling Hei \\ Mathematics Department \\ Hong Kong Bapist University \\ Hong Kong \\ E-mail: lightisgood2008@yahoo.cn
}

\begin{abstract}
In this paper, We study and prove the existence of blow-up solution of generalized $(2+1)$ dimensional Kaup-Kupershmidt (KK) equation by direct construction of an exact blow-up solution . Since Sawada-Kotera equation and Kaup-Kupershmidt (K. Sawada, 1974) equation are only particular case of our generalized equation, we can also prove the existence of blowup solution of Sawada-Kotera equation and Kaup-Kupershmidt (K. Sawada, 1974) equation by our construction of exact solution.
\end{abstract}

Keywords: Kaup—Kupershmidt Equation, Blow up, Exact solution

\section{Introduction}

The inverse scattering transform method is a powerful tool for the investigation of nonlinear differential equations (V.E. Zakharov, 1980; M.J. Ablowitz, 1981; F. Calogero, 1982).

The extent of applicability of this method crucially depends on the dimension of space. There are a great number of various nonlinear evolution equations in $1+1$ dimensions (one spatial and one time dimension) integrable by the inverse scattering transform method (V.E. Zakharov, 1980; M.J. Ablowitz, 1981; F. Calogero, 1982). For two spatial dimensions the class of integrable equations is much poorer. The best known two-dimensional integrable equations which have physical applications are the Kadomtsev-Petviashvili equation, three-wave equations and the Davey-Stewarson equation (V.E. Zakharov, 1980; M.J. Ablowitz, 1981).

In the present paper we consider a nonlinear evolution equation in two spatial dimensions $(x, y)$ connected with the differential operator

where $\partial x \equiv \partial / \partial x, \partial y \equiv \partial / \partial y, V 0(x, y, t), \ldots, V N(x, y, t)$ are scalar functions. The applicability of the inverse scattering transform method to the problem has been demonstrated in ref. (V.E. Zakharov, 1974).

We present here some nonlinear evolution equations in $2+1$ dimensions $(x, y, t)$ for one dependent variable $u(x, y, t)$ which can be represented as the commutativity condition $[\mathrm{L}, \mathrm{T}]=\mathrm{LT}-\mathrm{TL}=0$. The operator $\mathrm{L}$ is of the form (1). The operators $\mathrm{T}$ will be given explicitly. The obtained equations are the two dimensional generalizations of the well-known Gardner (combined KdV and modified KdV equation ) equation, the Sawada-Kotera, the Kaup-Kupershmidt and the Harry Dim equations.

Now we consider the operator $\mathrm{L}$ of the third order. The first example is

$$
\begin{gathered}
L=\partial_{x}^{3}+u \partial_{x}+\partial_{y} \\
T=9 \partial_{x}^{5}+15 u \partial_{x}^{3}+15 u_{x} \partial_{x}^{2}+\left(10 u_{x x}+5 u^{2}-5 \partial_{x}^{-1} u_{y}\right) \partial_{x}+\partial / \partial t
\end{gathered}
$$

The operators (1) and (2) commute if

$$
\partial u(x, y, t) / \partial t=u_{x x x x x}+5 u_{x} u_{x x}+5 u u_{x x x}+5 u^{2} u_{x}+5 u_{x x y}-5 \partial_{x}^{-1} u_{y y}+5 u u_{y}+5 u_{x} \partial_{x}^{-1} u_{y}
$$

(3) is the two-dimensional integrable generalization of the Sawada-Kotera equation (K. Sawada, 1974).

Another example is 


$$
L=\partial_{x}^{3}+u \partial_{x}+\frac{1}{2} u_{x}+\partial_{y}
$$

The corresponding evolution equation is

$$
\begin{aligned}
& T=9 \partial_{x}^{5}+15 u \partial_{x}^{3}+\frac{45}{2} u_{x} \partial_{x}^{2}+\left(\frac{35}{2} u_{x x}+5 u^{2}-5 \partial_{x}^{-1} u_{y}\right) \partial_{x}+\left(5 u u_{x}-\frac{5}{2} u_{y}+5 u_{x x x}\right)+\partial / \partial t \\
& \partial u(x, y, t) / \partial t=u_{x x x x x}+5 u u_{x x x}+\frac{25}{2} u_{x} u_{x x}+5 u^{2} u_{x}+5 u_{x x y}-5 \partial_{x}^{-1} u_{y y}+5 u u_{y}+5 u_{x} \partial_{x}^{-1} u_{y}
\end{aligned}
$$

(6)is the equation which was considered by Kaup and Kupershmidt (D.J. Kaup, 1980).

Let us emphasize that the parts of the two-dimensional Sawada-Kotera and Kaup-Kupershmidt equations (3) and (4) which contain the terms with derivative $\partial_{y}$ coincide. It is also easily seen that eqs. (3), (9), (11) can be represented in local form by intraducing the potential $W\left(u=W_{x}\right)$.

All equations considered have infinite series of the integrals of motion. They can be obtained by a standard recursion procedure if one represents the solution $\Psi$ of the equation $L_{\Psi}=0$ in the form

$$
\Psi=\exp \left(i \lambda x+i \lambda^{N} y+\partial_{x}^{-1} x(x, y, t, \lambda)\right)
$$

(see refs. V.E. Zakharov, 1980; M.J. Ablowitz , 1981; D.J. Kaup, 1980)

Similar to their one-dimensional limit the two-dimensional equations (3), (4), (7), (9), (11) may have various physical applications.

\section{Main Result}

Now, we can introduce the (2+1) dimensional generalized Kaup-Kupershmidt equation (B. Konopelchenko, 1984; M.J. Ablowitz, 1991; S.B. Leble, 1994):

$$
a u_{t}+b u_{x x x x x}+c u u_{x x x}+d u_{x} u_{x x}+e u^{2} u_{x}+f u_{x x y}+g\left(\partial_{x}^{-1} u_{y}\right)=0
$$

Where a, b, c, d, e, f, g, h, i are all nonzero constant. Sawada-Kotera equation

$$
\partial(x, y, t) . \partial t=u_{x x x x x}+5 u_{x} u_{x x}+5 u u_{x x x}+5 u^{2} u_{x}+5 u_{x x y}-5 \partial_{x}^{-1} u_{y y}+5 u u_{y}+5 u_{x} \partial_{x}^{-1} u_{y}=0
$$

and Kaup-Kupershmidt equation

$$
\partial u(x, y, t) / \partial t=u_{x x x x x}+5 u u_{x x x}+\frac{25}{2} u_{x} u_{x x}+5 u^{2} u_{x}+5 u_{x x y}-5 \partial_{x}^{-1} u_{y y}+5 u u_{y}+5 u_{x} \partial_{x}^{-1} u_{y}=0
$$

are only special form of the equation (11)

$$
a u_{t}+b u_{x x x x x}+c u u_{x x x}+d u_{x} u_{x x}+e u^{2} u_{x}+f u_{x x y}+g\left(\partial_{x}^{-1} u_{y y}\right)+h u u_{y}+i u_{x}\left(\partial_{x}^{-1} u_{y}\right)=0
$$

In (Xing-Biao $\mathrm{Hu}, 1999)$, A bilinear form for the equation is found and

then 3-soliton solutions are obtained with the assistance of software Mathematica. Six symmetries of the bilinear $2+1$ dimensional KK equation are given and their symmetry algebra is identified. But up to now, no one consider the general case (11).

(11) is a nonlinear, fifth order, 3-dimentional, differential integral equation. It is extremely difficult. To our knowledge, very little is known for this equation. However, we still successfully construct an analytic exact solution for it. Our solution is a blowing up solution. The solution will blow up in the neighborhood of the plane $p x+q y+r t+s=0$. The operator $\partial_{x}^{-1}$ is the inverse operator of $\partial_{x}, \partial_{x}^{-1}$ is an partial integration operator, and $\partial_{x} \partial_{x}^{-1}=\partial_{x}^{-1} \partial_{x}=1$

We assume the KK (12) equation has the blowing up solution of the form:

$$
u=A(p x+q y+r t+s)-2+B
$$


Where A, B, p, q, r, s, are the undetermined coefficient

Let $\zeta=p x+q y+r t+s$, then

$$
u=A \zeta^{-2}+B
$$

Substitute (12) into (11), then

$-2 a r A \zeta^{-3}+b p^{5} A(-2)(-3)(-4)(-5)(-6) \zeta^{-7}+c A^{2} p^{3}(-2)(-3)(-4) \zeta^{-7}+(-2)(-3)(-4) c B A p^{3} \zeta^{-5}+d(-2)^{2}(-3) A^{2} p^{3} \zeta^{-7}+$ $e\left(A^{2 \zeta^{-4}}+2 A B \zeta^{-2}+B^{2}\right) A p \zeta^{-3}(-2)+f A(-2)(-3)(-4) p^{2} q \zeta^{-5}+(-2) g p^{-1} q^{2} A \zeta^{-3}+h A^{2}(-2) q \zeta^{-5}+A h B(-2) q \zeta^{-3}+i(-2) A^{2} q \zeta^{-7} \zeta^{-5}=$ 0

That is:

$$
\begin{gathered}
{\left[-2 a r A-2 e A p B^{2}-2 g p^{-1} q^{2} A-2 h A B q\right] \zeta^{-3}+} \\
{\left[-24 c B A p^{3}-4 e A^{2} B p-24 f A p^{2} q-2 h A^{2} q-2 i A^{2} q\right] \zeta^{-5}+\left[-720 b p^{5} A-24 c A^{2} P^{3}-12 p^{3} d A^{2}-2 e A^{3} P\right] \zeta^{-7}=0}
\end{gathered}
$$

Equating both sides, we get

$$
\begin{gathered}
-2 a r A-2 e A p B^{2}-2 g p^{-1} q^{2} A-2 h A B q=0(16) \\
-24 c B A p^{3}-4 e A^{2} B p-24 f A p^{2} q-2 h A^{2} q-2 i A^{2} q=0(17) \\
-720 b p^{5} A-24 c A^{2} P^{3}-12 p^{3} d A^{2}-2 e A^{3} P=0
\end{gathered}
$$

Now, we have only 3 equations as restrictions, but we have 5 free variables $(\mathrm{A}, \mathrm{B}, \mathrm{p}, \mathrm{q}, \mathrm{r})$, so there are infinitely many solutions. Now we regard $\mathrm{p}, \mathrm{q}$ as free parameters, A, B, $\mathrm{r}$ as variables, to solve (13).

$$
\begin{aligned}
A & =\frac{-12 c p^{2}-6 p^{2} d \pm \sqrt{\left(12 c p^{2}+6 p^{2} d^{2}\right)-1440 b p^{4}}}{2 e} \\
B & =\frac{-i A q-h A q-12 f p^{2} q}{12 c p^{3}+2 e A p} \\
r & =\frac{-e p B^{2}-g p^{-1} q^{2}-h B q}{a}
\end{aligned}
$$

Where $\mathrm{p}, \mathrm{q}$ are free parameters.

If $\mathrm{e}=0$,

$$
A=-\frac{2 c p^{2}+p^{2} d}{60 b p^{4}}
$$

In the above solution of algebra equation. We firstly express A in terms of free parameter and coefficient, then express B in terms of $A$ and free parameter and coefficient, and express $r$ in terms of B and free parameter and coefficient, in this way we express A, B, $\mathrm{r}$ in terms of free parameter and coefficient. What's more, at first, we assume all coefficient are non-zero, but in the process we find that we can still manage to find the solution even if e is zero, therefore, the requirement of keeping e to be non-zero constant can be removed. And we should notice that the solution is complex when

$$
e \neq 0
$$

and

$$
\left(12 c p^{2}+6 p^{2} d^{2}\right)-1440 b p^{4}<0
$$

and the solution is real when $\mathrm{e}=0$ 


\section{Concluding Remarks}

(1) Since Sawada-Kotera equation (K. Sawada, 1974) and Kaup-Kupershmidt (D.J. Kaup, 1980) equation and Hu-WangQian equation (11) are only particular case of our generalized equation (12), we can also prove the existence of blow-up phenomenon of Sawada-Kotera equation and Kaup-Kupershmidt (D.J. Kaup, 1980) equation by our construction of exact solution and $\mathrm{Hu}-$ Wang-Qian equation (Xing-Biao $\mathrm{Hu}, 1999$ ).

(2) Note that A has two values so there is a bifurcation in A. The solution has two branch, not single one.

In conclusion, we prove that Generalized Kaup-Kupershmidt equation (11) has a blowing up solution for all choices of nonzero parameters a , b, c, d, e, f, g, h, i. The solution will blow up in the neighborhood of the plane

$$
p x+q y+r t+s=0
$$

\section{References}

B.G. Konopelchenko. (1982). Phys. Lett., 92A, 323.

B. Konopelchenko. (1984). V.Dubrovsky. Physics Letters A, 102,15.

F. Calogero and A. Degasperis. (1982). Spectral transform and solitons, Vol. 1 (North-Holland, Amsterdam).

K. Sawada and J. Kotera. (1974). Prog. Theor. Phys., 51, 1355. D.J.

Kaup, Stud. (1980). Appl. Math. 62, 189.

M.J. Ablowitz, P.A. Clarkson, Solitons. (1991). Nonlinear Evolution Equations and Inverse Scattering, Cambridge, Cambridge University Press.

M.J. Ablowitz and H. Segur. (1981). Solitons and the inverse scattering transform (SIAM, Philadelphia).

S.B. Leble, N.V. Ustinov. (1994). Inverse Problems, 10, 617.

V.E. Zakharov, S.V. Manakov, S.P. Novikov and L.P. Pitaevsky. (1980). Theory of solitons. Method of inverse problem (Nauka, Moscow).

V.E. Zakharov and A.B. Shabat. (1974). Funct. Anal. Appl., 843.

Xing-Biao Hu, Dao-Liu Wang, Xian-Min Qian. (1999). Soliton solutions and symmetries of the $2+1$ dimensional KaupKupershmidt equation Physics Letters A, 262, 409-415.

\section{$==ニ==$
NOTICE}

Dear Readers,

Here, we would like to claim that, we haven't updated the latest print version of the paper entitled "Integral Oscillation Criteria for Second-Order Linear neutral Delay Dynamic Equations on Time Scales" published in the issue of Vol. 2, No. 1, February 2010. We have updated this paper on the website. We apologize for any inconvenience.

Editorial Board of Journal Mathmatics Research

May 1, 2010 